Fußnetz Bayern e.V.

\title{
Bessere Versorgung für diabetische Füße
}

\author{
Die Zahlen sind erschreckend: Alljährlich wird in Deutschland $62000 \mathrm{mal}$ amputiert, alle \\ 19 Minuten diabetesbedingt [1] . Das Fußnetz Bayern e.V. möchte dazu beitragen, diese \\ Zahl durch eine bessere Versorgung der Fußpatienten und zum Wohle aller zu senken.
}

Für viele Patienten sind die Folgen der Amputation fatal: Mehr als ein Drittel der Diabetiker stirbt ein Jahr nach der Amputation. Bei 50\% der Beinamputierten musste innerhalb der nächsten vier Jahre auch am anderen Bein amputiert werden. Die Reha-Maßnahmen und die Hilfsmittel sind mit hohen Kosten für Gesundheitssystem und Rentenkassen verbunden.

Eine der Ursachen für die hohen Amputationszahlen in Deutschland ist, dass für die Therapie bei Diabetischen Fußsyndrom (DFS) viele Spezialisten nötig, aber oft nicht verfügbar sind. Hinzu kommt, dass viele niedergelassene Hausärzte und Internisten nicht ausreichend in der DFS-Therapie ausgebildet sind und eine gute ambulante Versorgung oft schon an den Abrechnungsmodalitäten des Gesundheitssystems scheitert. So mangelt es - wie die Kasuistik auf Seite 6 und 7 zeigt - auch oft an der hinreichenden Entlastung des Fußes, die zwingend nötig ist. Das ist kein böser Wille. Der Patient ist sich ja keines Schmerzes bewusst, der ihn am Belasten hindern würde. Jeder Schritt auf einer Wunde ist aber zu viel, zerstört das zarte Granulationsgewebe wieder. Darum ist es so wichtig, dass wir unsere Patienten mit Rat und Tat unterstützen.

Im Fußnetz Köln zählt der Total Contact Cast (TCC) zu einer mit Festpreis verankerten wertvollen Therapieoption. Sonst wird diese Methode den orthopädischen Schuhmachern nicht mehr bezahlt, sondern den Ärzten als ,intrabudgetär“ und ärztliche Maßnahme zugeordnet. Dies hat zur Folge, dass Pflegediensten der TCC-Wechsel nicht mehr erstattet wird. Patienten müssten viel öfter in die Praxis kommen, was zu einer vermehrten Einweisungsrate mit erheblichen Kosten für das Gesundheitssystem führt.

Mit abgestimmten Behandlungskonzepten, gemeinsamer Betreuung der Patienten und intensivem kollegialen Austausch will auch das Fußnetz Bayern e.V. eine hochwertige, flächendeckende und wirtschaftliche Versorgung erzielen. Dazu sind intensive Gespräche mit den Krankenkassen nötig. Diese sind zwar grundsätzlich an einer besseren Fußversorgung ihrer Mitglieder interessiert, halten sich aber wegen des Gesundheitsfonds mit Vertragsabschlüssen zurück. In der Region München läuft allerdings schon seit über einem Jahr mit der KKH-Allianz ein integriertes Versorgungsmodell sehr erfolgreich.

Die Ziele des Fußnetzes Bayern e.V.: Durch eine frühzeitige optimale Therapie möglichst viele Amputationen bei Diabetikern verhindern und mittelfristig auch dieTherapie- und Folgekosten senken. Die Hürden will das Fußnetz mit beharrlichen, konstruktiven Verhandlungen nehmen. Es ist ein Zusammenschluss von über 150 Fachärzten und Spezialisten, der nach dem Vorbild des Kölner Netzwerks die Versorgung von DFS-Betroffenen optimieren will.

Infos: www.fussnetz-bayern.de oder www.fkdb.net. 1. Liebl A et al. DMW 2001;126(20): 585-9.

\section{Dr. med. Arthur Grünerbel}

Bertelestrasse 18 und Diabeteszentrum München Süd, Herterichstr. 51, D-81479 München

gruenerbel@diabeteszentrum-muenchen-sued.de

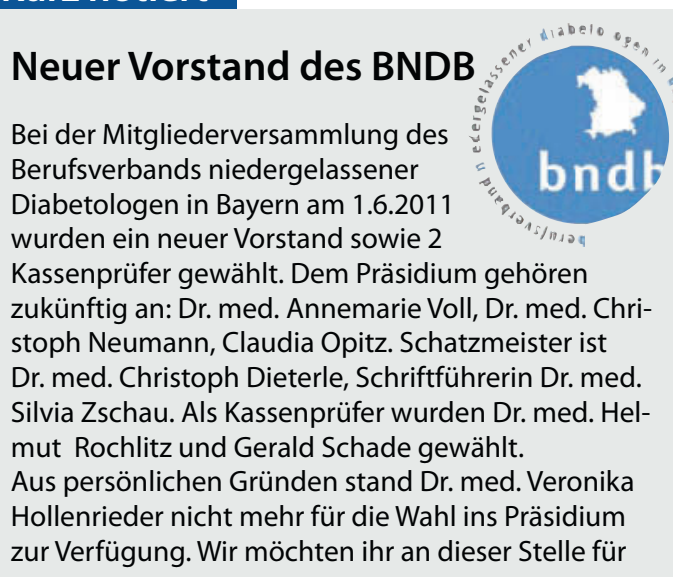

ihr unermüdliches Engagement und ihre hervorragende Arbeit von ganzem Herzen danken. Die Ziele des neu gewählten Präsidiums sind wie folgt: Verbesserung der Kommunikation der Mitglieder durch vereinfachte Benutzung der Website durch Erstellung eines neuen Moduls; Vorstellung des Präsidiums bei GKV und KV; nachhaltige Modifikation des DMP-Plattformvertrages zum Erhalt der flächendeckenden Versorgung mit Schwerpunktpraxen, Übergangsregelungen für nicht ärztliches Personal; Weiterentwicklung der Diabetesverträge; Entwicklung von Zusatz/Selektivverträgen

Herzliche Grüße vom Präsidium
A. Voll
C. Neumann
C. Opitz 